\title{
Estudio comparativo de las técnicas de extrusión y prensado como procesos de conformado de productos cerámicos de construcción en el Área Metropolitana de Cúcuta
}

\author{
J. F. GELVES ${ }^{1}$, R. MONROY ${ }^{1}$, J. SÁNCHEZ ${ }^{1}$, R. P. RAMIREZ ${ }^{1}$ \\ ${ }^{1}$ Centro de Investigación de Materiales Cerámicos, Grupo de Investigación en Tecnología Cerámica GITEC, \\ Universidad Francisco de Paula Santander, Cúcuta-Colombia.
}

\begin{abstract}
El presente trabajo estudia las principales variables de control del proceso de fabricación de piezas cerámicas de construcción de la zona metropolitana de San José de Cúcuta, mediante las técnicas de extrusión y prensado para su conformado. La investigación se llevó a cabo utilizando muestras arcillosas de las dos principales formaciones geológicas de la región de donde se extraen las materias primas para el proceso a nivel industrial. Las muestras arcillosas se molturaron tanto por vía seca como por vía húmeda y se estudió su granulometría. Posteriormente se llevó a cabo el proceso de conformado utilizando una prensa hidráulica y una extrusora con vacío, ambos equipos a escala de laboratorio. Las probetas obtenidas se secaron y cocieron entre $980^{\circ} \mathrm{C}$ y $1180^{\circ} \mathrm{C}$ al final del proceso se realizaron pruebas para determinar la absorción de agua, contracción y pérdida de masa en cocción de las probetas. Los resultados de la investigación dejaron ver que la técnica de extrusión permite una gresificación más rápida para las arcillas de la región en comparación con la técnica de prensado, las contracciones de secado y cocción son menos marcadas en la técnica de prensado y con desviaciones estándar mucho mas bajas que en el caso de la extrusión.
\end{abstract}

Palabras clave: Extrusión, prensado, conformado, absorción de agua, cerámica roja.

The comparative study of pressing and extrusion like procesess of construction ceramic products in the Metropolitan Area of Cucuta

The present work studies the principal variables of control in the manufacturing process of construction pieces of the Metropolitan Area of San Jose de Cucuta by extrusion and pressing techniques for its forming. The investigation was taken out using clayey samples of the two principal geological formations of the region where the raw material is taken for processing at an industrial level. The clayey samples milling was made by dry means as well as by moisture means and its particle size was measured. Subsequently the forming process was taken over by using an hydraulic press and extruder with vacuum system, both equipments at laboratory scale, the pieces shaped were dry and firing between $980^{\circ} \mathrm{c}$ and $1180^{\circ}$ at the end of the process the tests were made to determine water absorbtion, contraction and mass loss at the pieces firing. The study results left to see that the extrusion technique allowed a faster vitrification for the region's clay in comparing with the pressing technique, the contractions of drying and firing are less marked on the pressing techniques with standard deviations much lower than in extrusion.

Key words Extrusion, pressing, shaped, absorption of water, Red ceramics.

\section{INTRODUCCIÓN}

El área metropolitana de Cúcuta se caracteriza por la gran concentración de empresas dedicadas a la extracción y transformación de la arcilla en productos cerámicos de construcción; la región posee un gran potencial de materias primas arcillosas concentradas mayoritariamente en la formación geológica León y el grupo Guayabo. Las arcillas provenientes de la formación León se caracterizan por su aspecto grisáceo mientras que las arcillas del grupo Guayabo presentan tonalidades más rojizas, cada una de estas materias primas posee un comportamiento en particular durante su aplicación en los procesos de fabricación de piezas cerámicas de construcción (1).
La industria cerámica regional se caracteriza por el uso de las técnicas de prensado y extrusión para el conformado de las diferentes piezas elaboradas, siendo esta ultima técnica la de mayor frecuencia de trabajo en la región (2). Aunque los aspectos monetarios son de gran influencia a la hora de elegir el sistema productivo y tecnológico a emplear, la naturaleza de las materias primas empleadas juega un papel muy importante en la calidad de los productos elaborados y en el éxito financiero de la unidad productiva implementada (3).

Los empresarios de la región dedicados a esta industria han conformado sus empresas sin tener claridad de cuál es el método que mejor se adapta a sus intereses (4), esto se debe 
a la falta de referentes bibliográficos de consulta. La presente investigación se ha realizado como un aporte a esta problemática tanto para los empresarios como para las unidades académicas e investigativas que guardan relación con el tema.

\section{PARTE EXPERIMENTAL}

\subsection{Materias primas}

La investigación fue desarrollada en el área metropolitana de San José de Cúcuta, Norte de Santander. El muestreo utilizado fue de tipo intencionado, la muestra correspondiente al grupo Guayabo se obtuvo de la Mina Chía, ubicada en jurisdicción del municipio del El Zulia y la muestra de la formación León se obtuvo de la mina Peracos, ubicada al norte de la ciudad de Cúcuta en la zona circundante al cerro Tasajero; La investigación realizada es de tipo exploratoria y experimental la cual se llevó a cabo en las instalaciones del Centro de Investigación de Materiales Cerámicos CIMAC. El análisis químico, fue realizado por FERRO CORPORATION utilizando Fluorescencia de Rayos X (5), los cuales se presentan en la tabla I. A su vez se pueden observar en la figura 1 y 2 , los análisis mineralógicos de las dos arcillas realizado por la Universidad Industrial de Santander aplicando la técnica de Difracción de Rayos X (DRX) (6). De forma complementaria en la tabla II se muestra los resultados del análisis del tamaño de partícula de estos suelos, proceso que se desarrolló bajo la técnica del hidrómetro (6), estos aspectos son básicos para el establecimiento de la aptitud cerámica de las materias primas.

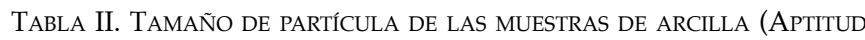
CERÁMICA)

\begin{tabular}{|c|c|c|c|c|c|}
\hline \multicolumn{2}{|c|}{ Muestra de Arcilla Roja } & \multicolumn{3}{c|}{ Muestra de Arcilla Gris } \\
\hline \% Arcilla & $\%$ Limos & $\%$ Arenas & $\%$ Arcilla & $\%$ Limos & $\%$ Arenas \\
\hline 55,2 & 31,4 & 13,4 & 67,1 & 29,3 & 3,6 \\
\hline
\end{tabular}

TABLA I. ANÁLISIS QUímICO DE LAS MUESTRAS ARCILLOSAS EMPLEADAS

\begin{tabular}{|c|c|c|c|c|c|c|c|c|}
\hline MUESTRA (\%) & $\mathrm{SiO}_{2}$ & $\mathrm{Al}_{2} \mathbf{O}_{3}$ & $\mathrm{TiO}_{2}$ & $\mathrm{Fe}_{2} \mathbf{O}_{3}$ & $\mathrm{CaO}$ & $\mathbf{M g O}$ & $\mathbf{K}_{2} \mathbf{O}$ & $\mathbf{N a}_{2} \mathbf{O}$ \\
\hline Arcilla Roja (Guayabo) & 66,6096 & 23,6559 & 0,9844 & 5,8045 & 0,5551 & 0,5171 & 1,7162 & 0,1572 \\
\hline Arcilla Gris (León) & 67,08 & 24,29 & 0,88 & 5,21 & 0,43 & 0,52 & 1,14 & 0,45 \\
\hline
\end{tabular}

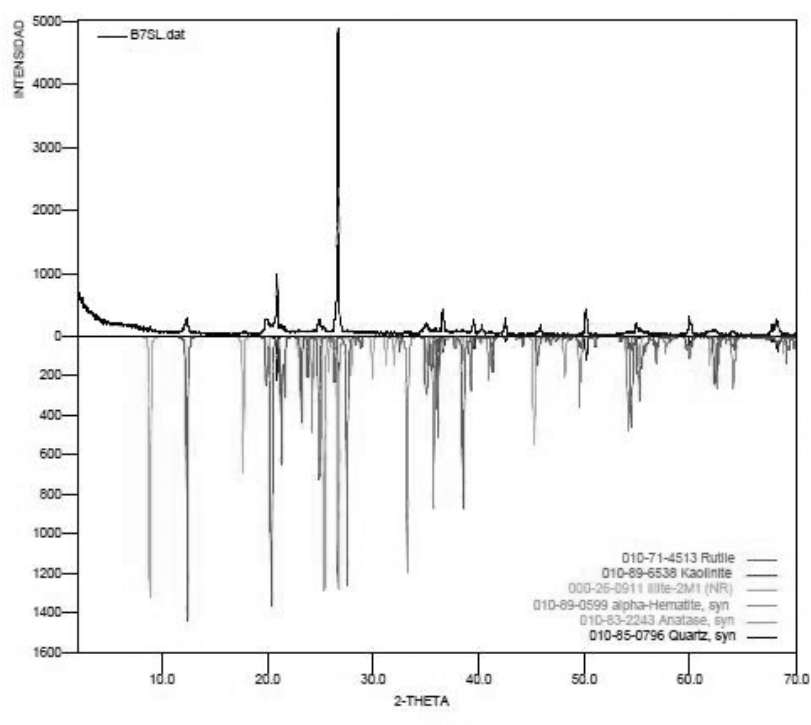

Fases Cristalinas encontradas en la muestra original.

\begin{tabular}{|c|c|c|c|}
\hline \multicolumn{1}{|c|}{ FASE } & $\begin{array}{c}\text { No. TARJETA } \\
\text { PDF-2 }\end{array}$ & NOMBRE \\
\hline \multirow{4}{*}{ Cristalinos } & $\mathrm{Si} \mathrm{O}_{2}$ & $010-85-0796$ & Cuarzo \\
\cline { 2 - 4 } & $\mathrm{Fe}_{2} \mathrm{O}_{3}$ & $010-89-0599$ & Hematita \\
\cline { 2 - 4 } & $\mathrm{Al}_{2} \mathrm{Si}_{2} \mathrm{O}_{5}(\mathrm{OH})_{4}$ & $010-89-6538$ & Caolinita \\
\cline { 2 - 4 } & $\mathrm{Ti} \mathrm{O}_{2}$ & $010-83-2243$ & Anatasa \\
\cline { 2 - 4 } & $\mathrm{Ti} \mathrm{O}_{2}$ & $010-71-4513$ & Rutilo \\
\cline { 2 - 4 } & $\left(\mathrm{K}, \mathrm{H}_{3} \mathrm{O}\right) \mathrm{Al}_{2} \mathrm{Si}_{3} \mathrm{Al} \mathrm{O}_{10}(\mathrm{OH})_{2}$ & $000-26-0911$ & illita \\
\hline
\end{tabular}

Figura 1. Análisis mineralógico de la arcilla roja (Formación Guayabo)
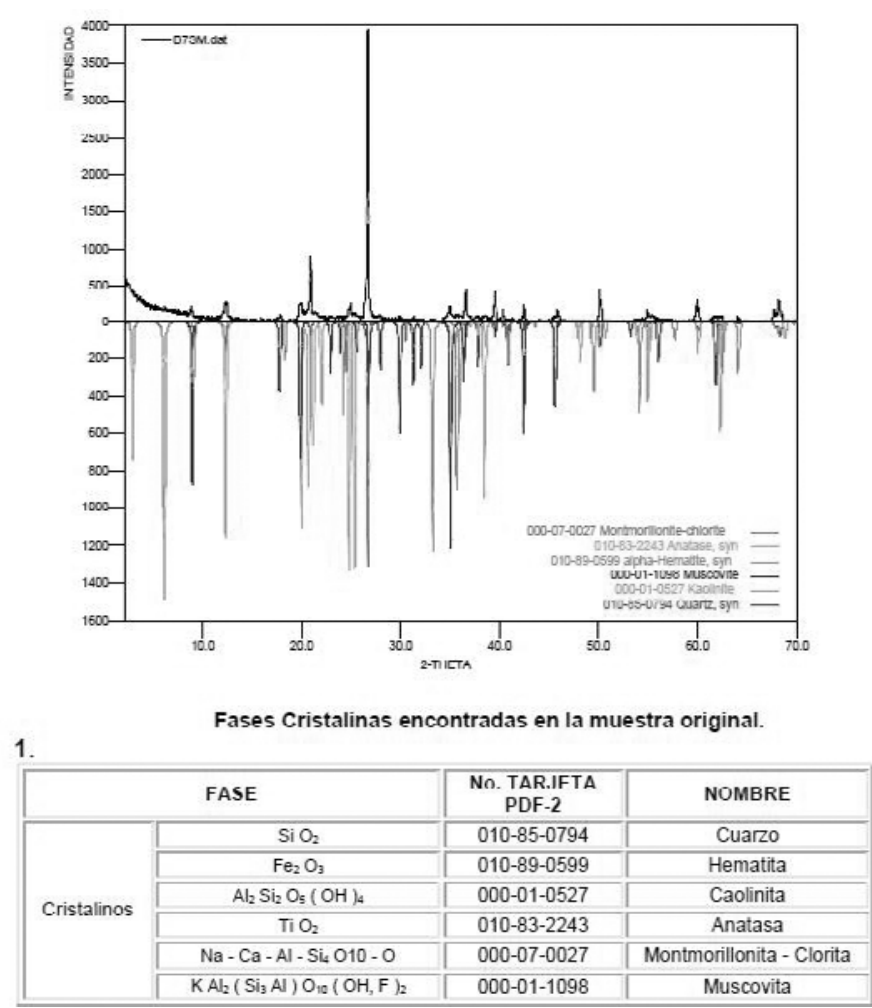

Figura 2. Análisis mineralógico de la arcilla gris (Formación León) 


\subsection{Equipo empleado}

Para la realización de la investigación se contó con una extrusora de laboratorio con vacío modelo NEW WAVE fabricada por la empresa Metal Souza Ltda., prensa de laboratorio de 40 toneladas, marca Gabbrielli y molde circular , molino de martillos de laboratorio marca Servitech modelo CT-058, equipo para molienda por vía húmeda a escala de laboratorio marca Gabbrielli, estufa de secado marca "Dies" cuyo calentamiento se hace con resistencia eléctrica, vibrotamiz, juego de tamices mallas ASTM 230, 200, 120, 60, 30 y 12, plasticimetro de Pfefferkorn, Equipo para medir densidad aparente de probetas cerámicas por inmersión en mercurio (mercurimetro) marca Gabbrielli, Horno mufla modelo 10/12 marca Gabbrielli, Balanzas digital marca OHAUS adventurer, termobalanza OHAUS MB45 y calibrador digital marca Mitutoyo. La figura 3 muestra el equipo empleado para la molienda y el conformado de las probetas.

\subsection{Elaboración de probetas}

Inicialmente los materiales recolectados fueron sometidos al proceso de reducción de tamaño de partícula, se molturaron por vía seca en el molino de martillos, y por vía húmeda en el equipo de molienda para tal fin, durante un tiempo de 2,0 minutos empleando alcohol industrial al $90 \%$ de pureza en lugar de agua, (el uso de este compuesto permite agilizar el proceso de secado sin afectar la granulometría de la arcilla molida ni las propiedades físicas ni químicas de la misma, el tiempo de molienda empleado fue establecido para obtener una distribución granulométrica uniforme que es una variable típica de control en las empresas que hacen molienda por vía húmeda en la región. El recipiente empleado tiene una capacidad de 1000,0 g, la mezcla contenía 400,0 g de material arcilloso, 400,0 g de alcohol y 200,0 g de bolas de alúmina de las cuales el $60 \%$ fueron bolas gruesas, $30 \%$ medianas y $20 \%$
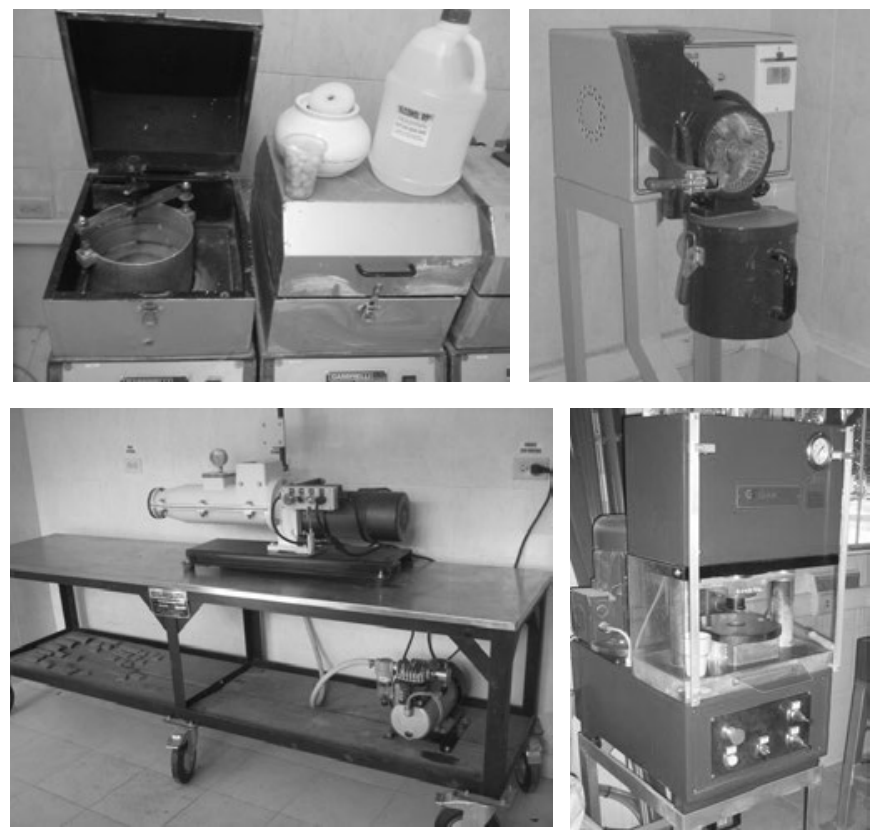

Figura 3. Equipo empleado para la molienda y el conformado de las probetas pequeñas). A el material molido por vía seca se le realizó un proceso de tamizado utilizando el tamiz malla 12 de uso común en la industria cerámica regional que utiliza este sistema de molienda, mientras que a la muestra obtenida por vía húmeda se le realizó lavado sobre tamiz malla 230 para identificar el porcentaje de retenido. Las muestras de arcilla roja y gris molturadas por los dos sistemas anteriormente nombrados se llevaron a la estufa de secado hasta alcanzar peso constante (7), se midió la granulometría de una muestra representativa de $200 \mathrm{~g}$ de cada material utilizando los tamices con luz de malla 30, 60,120, 200 y colector, en el vibrotamiz.

A continuación, se procedió a la preparación de las materias primas para los procesos de moldeo, las muestras molturadas en vía húmeda, requerían alcanzar una humedad del $6 \%$ (porcentaje común en procesos de prensado para elaboración de baldosas de cerámica roja) para lo cual se tuvo que utilizar un atomizador manual, el material humectado se dejo reposar por 6 horas y se tomo lectura de la humedad haciendo uso de la termobalanza, asegurando así el nivel adecuado de humedad, seguidamente el material se peso en muestras de 30,0 gramos y se llevaron al molde circular de la prensa hidráulica de laboratorio, donde se aplicó una presión de 30 bares. En las muestras molturadas por vía seca se determinó el índice de plasticidad Pfefferkorn. (Esta técnica consiste en determinar la plasticidad de una arcilla por la compresión que experimenta una muestra en forma cilíndrica, de dimensiones determinadas con diferentes contenidos de agua, cuando se la golpea con un martinete o martillo pilón, de un peso determinado y que cae por su propio peso, recorriendo una trayectoria fija) (8). Los resultados del índice de plasticidad Pfefferkorn se presentan en la tabla III.

TABLA III. ÍNDICES DE PLASTICIDAD DE LAS MUESTRAS ARCILLOSAS UTILIZADAS

\begin{tabular}{|c|c|}
\hline Tipo de muestra & Índice de plasticidad \% \\
\hline Arcilla roja, Grupo Guayabo & 25,0 \\
\hline Arcilla gris, Formación León & 29,5 \\
\hline
\end{tabular}

De acuerdo con el resultado obtenido del índice de plasticidad, se humectaron manualmente las arcillas estudiadas y se mantuvieron durante 12 horas para conseguir una buena homogeneización. Posteriormente se extrusionaron las pastas cerámicas obtenidas y se obtuvieron probetas de geometría cuadrada de $4,0 \mathrm{~cm}$ de longitud. En la figura 4 se muestra un registro fotográfico del proceso de conformado de las probetas.
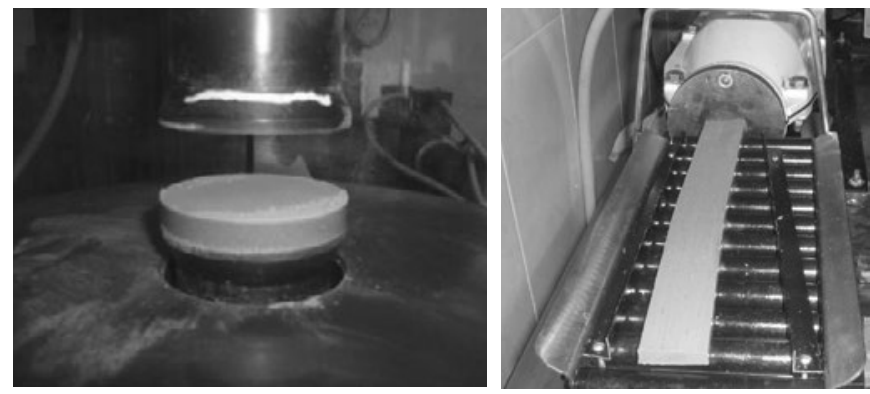

Figura 4. Registro fotográfico del proceso de conformado de las probetas 
Las probetas conformadas (cuatro probetas para cada temperatura de quema) se secaron en estufa de secado de resistencia eléctrica comenzando a temperatura ambiente $\left(30^{\circ} \mathrm{C}\right)$, durante las primeras cinco horas del proceso se realizaron incrementos de temperatura de $10{ }^{\circ} \mathrm{C} /$ hora, finalmente se llevo a la temperatura de $100{ }^{\circ} \mathrm{C}$ hasta completar 24 horas de secado. Una vez alcanzaron la temperatura ambiente, las probetas se pesaron y midieron: el diámetro para las probetas redondas y el lado para las probetas cuadradas.

Las probetas secas se llevaron a un horno mufla con calentamiento eléctrico, sometiendo las probetas a temperaturas de cocción en el rango comprendido entre los $980^{\circ} \mathrm{C}$ y $1180^{\circ} \mathrm{C}$ aumentado la temperatura en gradientes de $20^{\circ} \mathrm{C}$. En la figura 5 se pueden apreciar la curva de cocción empleada para el tratamiento térmico aplicado al material.

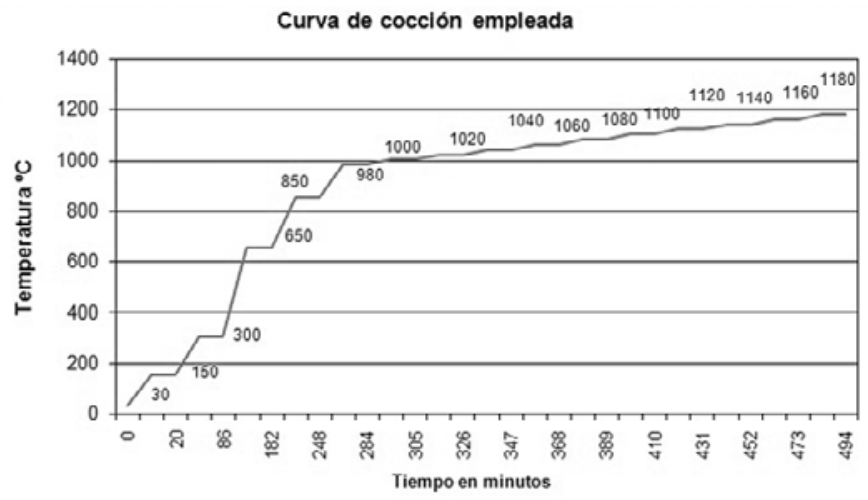

Figura 5. Curva de cocción empleada

Después del ciclo de cocción, cada lote de probetas se pesaron y midieron, una vez alcanzaron la temperatura ambiente. Finalmente se realizó la prueba de absorción de agua por ebullición, utilizando la metodología establecida en la norma técnica colombiana NTC 4321-3, (9). En la figura 6 se puede apreciar un diagrama secuencial del proceso realizado.

\section{RESULTADOS Y DISCUSIÓN}

\subsection{Diferencias granulométricas}

Las diferencias granulométricas entre la molienda en húmedo utilizada en la técnica de conformado por prensa, y la molienda en seco, de uso común en el conformado por extrusión se muestra en la figura 7.

Se aprecia que la molturación por vía húmeda permite una mayor proporción de partículas finas, caso contrario ocurre con la molturación por vía seca donde las partículas de mayor grano son las de mayor representatividad.

\subsection{Contracción de secado y cocción}

En la figura 8 se muestran las diferencias de contracción de secado presentadas en las probetas conformadas mediante las técnicas de extrusión y prensado, tanto para las arcillas rojas y grises; de igual manera en la figura 9 se presentan los cambios de contracción de cocción de dichas probetas en las diferentes temperaturas propuestas, los cálculos se realizaron basados en la norma ASTM (10).

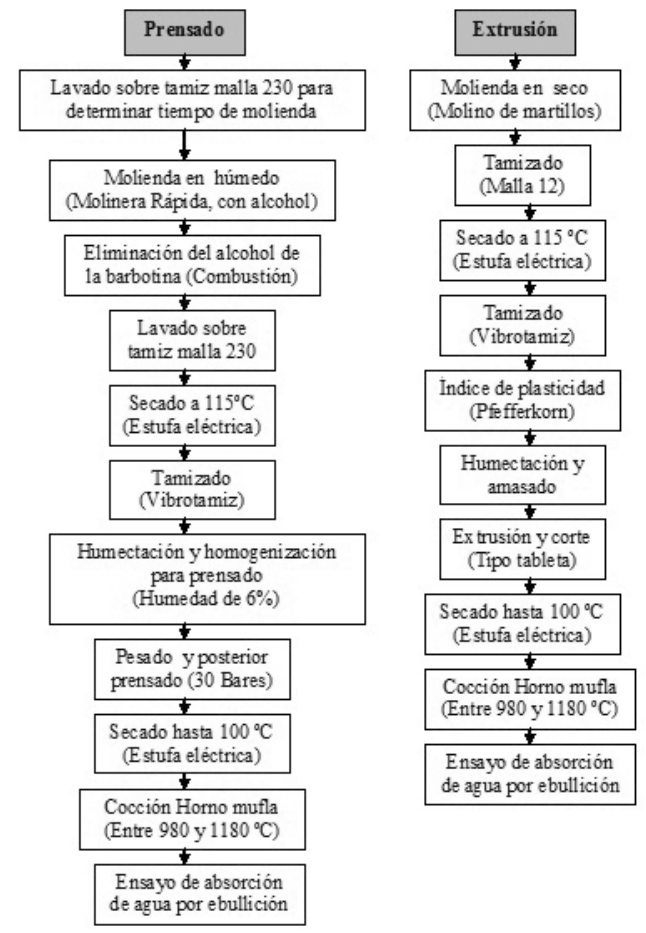

Figura 6. Esquema de la metodología aplicada

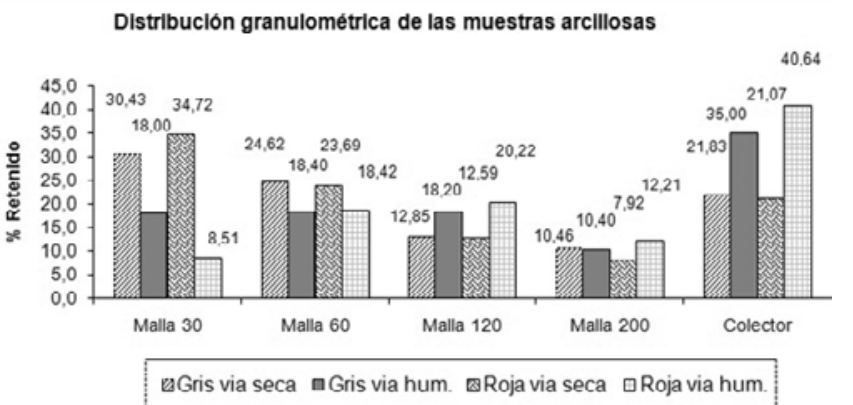

Figura 7. Distribución granulométrica de las arcillas molturadas

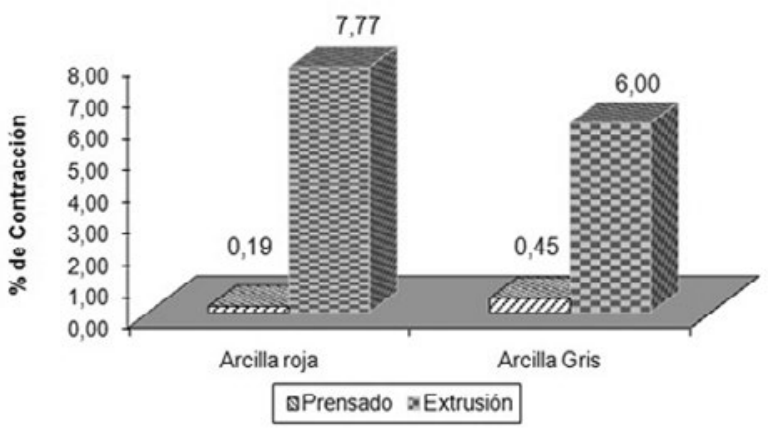

Figura 8. Contracciones de secado de las probetas conformadas por las técnicas de prensado y extrusión 
La figura anterior deja ver diferencias significativas de contracción de secado entre una técnica y otra, la técnica de extrusión presenta valores muy elevados, sin embargo, se aprecia que estas son mucho mas marcadas en la arcilla roja. Las arcillas rojas presentan mejor comportamiento utilizando la técnica de prensado, mientras que a las arcillas grises les va mejor en la extrusión. La desviación estándar en esta etapa del proceso cerámico es mucho mayor para la arcilla roja en la técnica de extrusión, con un $0,46 \%$, en la arcilla gris solo fue de $0,15 \%$; en referencia a la técnica de prensado los valores obtenidos fueron de $0,09 \%$ para la arcilla gris y $0,04 \%$ para la arcilla roja, contrario a lo obtenido en la técnica de extrusión.

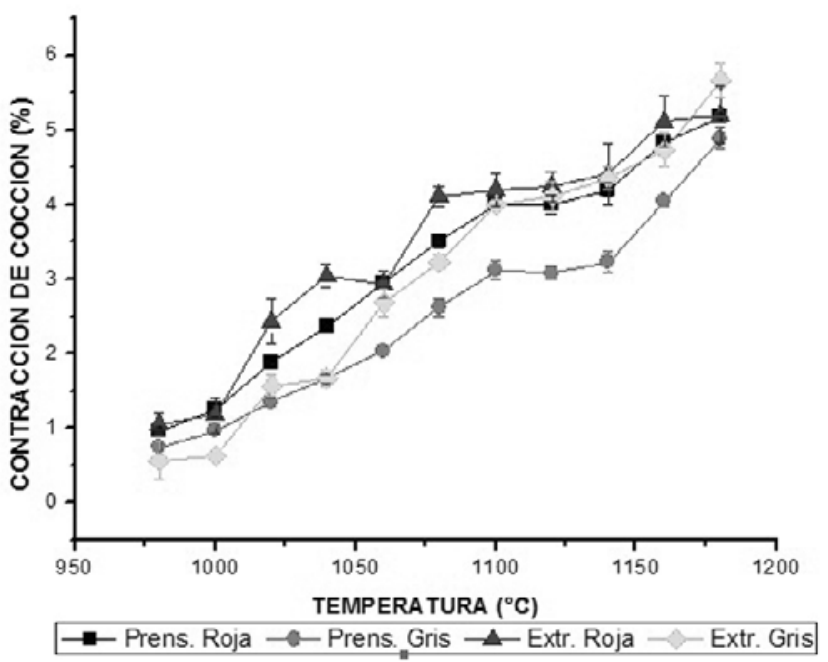

Figura 9. Contracciones de cocción de las probetas conformadas por las técnicas de prensado y extrusión

Se aprecia que la arcilla gris presenta unas contracciones mas bajas en comparación con la arcilla roja para las dos técnicas de conformado analizadas, aun siendo la muestra de la formación León mas plástica, la mayor presencia de alúmina en la arcilla gris juega un papel importante en esta variable, debido a la refractariedad de este compuesto. De los dos métodos analizados el prensado permite obtener contracciones mas bajas.

\subsection{Perdidas de calcinación y absorción de agua}

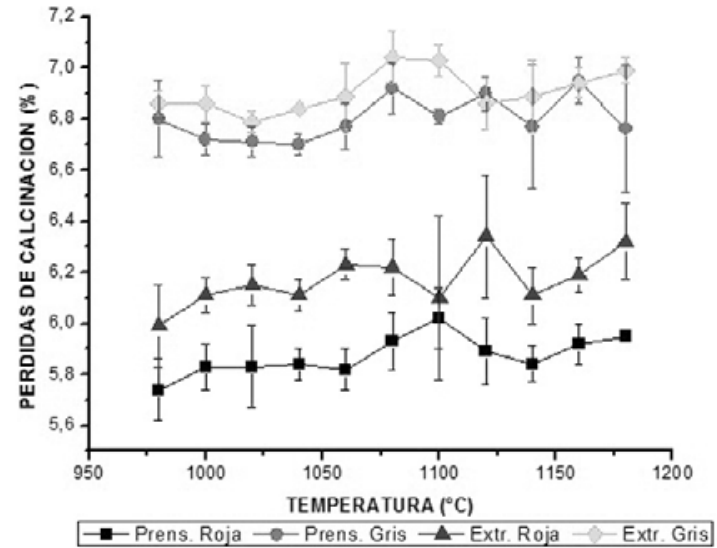

Figura 10. Perdidas de calcinación de las probetas conformadas por las técnicas de prensado y extrusión
Se observa que la técnica de prensado permite obtener unas perdidas de calcinación mas bajas que la extrusión, sin embargo las diferencias no tienen gran representatividad, la causa de este resultado puede encontrarse en el grado de compactación de las probetas, debido a la granulometría de la arcilla, que para el caso de la arcilla roja permite obtener una mayor porosidad facilitando la evaporación y salida del agua durante la etapa de secado.

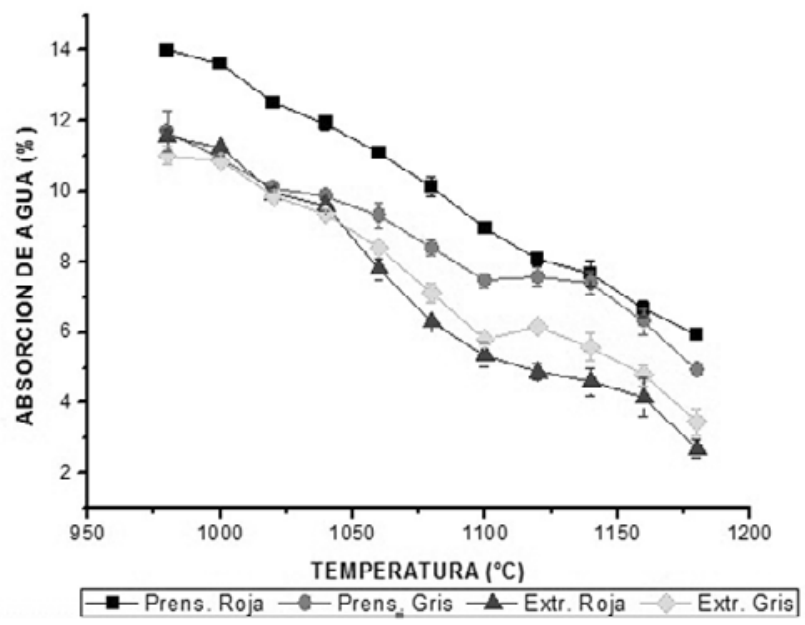

Figura 11. Absorción de agua de las probetas conformadas por las técnicas de prensado y extrusión

La figura deja ver que la técnica de extrusión permite obtener una menor porosidad de las piezas, con diferencias marcadas después de los $1040^{\circ} \mathrm{C}$, para la arcilla roja se evidencia que el efecto es mucho mayor que en la arcilla gris. Como ejemplo, en el caso de la temperatura de $1180^{\circ} \mathrm{C}$ las probetas de arcilla roja y gris conformadas por prensado presentaron una absorción de $5.95 \%$ y $4.94 \%$ respectivamente, para las del conformado por extrusión fue de $2.68 \%$ y $3.65 \%$.

\subsection{Densidad aparente de piezas cocidas}

La importancia de la Densidad Aparente (DAP) radica en el hecho de estar directamente relacionada con la microestructura interna de la pieza y determina, en gran medida, las propiedades del producto final (dimensiones, curvatura, resistencia a la helada, resistencia mecánica, presencia de corazón negro, acabado superficial, etc.) (11).

El modelo tradicional de mercurio es un modelo sencillo, el cual para el cálculo del DAP involucra dos variables de carácter cuantitativo: masa, empuje y la constante de densidad del Mercurio, dada por el valor de $13.650 \mathrm{Kg} /$ $\mathrm{m}^{3}$ (12), de acuerdo a esta técnica se calculo la densidad aparente de las muestras estudiadas, las cuales se observan en la figura 12. 


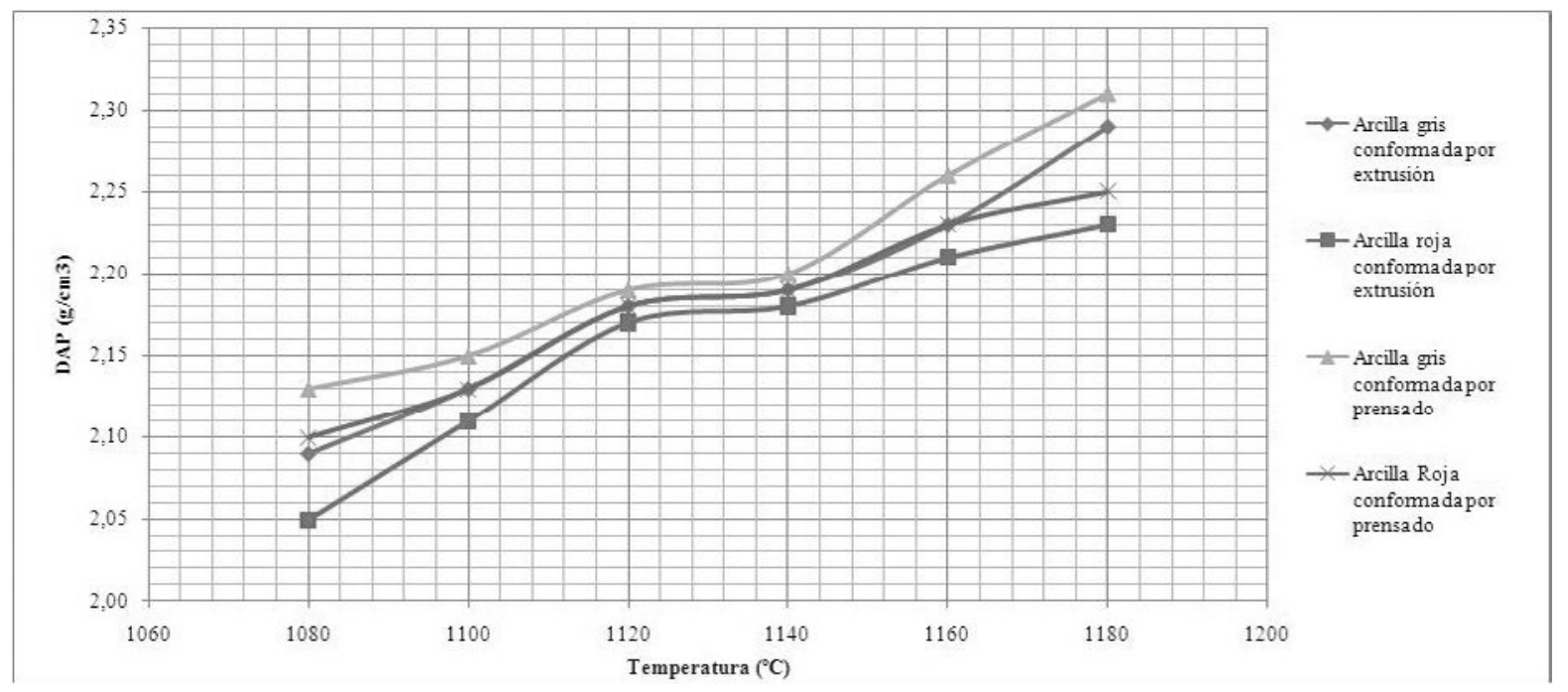

Figura 12. Densidad aparente de las probetas cocidas analizadas

En la figura 12 se observa que tanto en las arcillas rojas como grises conformadas por la técnica de extrusión presentan una menor densidad aparente comparada con la técnica de prensado. Estas diferencias son debidas al menor grado de empaquetamiento que se consigue con el proceso de conformado por extrusión comparado con el de prensado (13), puesto que la presión que se aplica en el prensado ha sido mayor que en la extrusión.

\section{CONCLUSIONES}

Al comparar las dos técnicas de conformado analizadas no se puede afirmar que exista una mejor técnica para ser utilizada en la fabricación de piezas cerámicas de construcción en el área metropolitana de Cúcuta, puesto que cada método ofrece unas ventajas particulares. En referencia al aspecto relacionado con las contracciones de secado de las piezas, se estableció que la técnica de prensado permite obtener valores más bajos y con una menor dispersión de datos que la extrusión, esta técnica sin duda será la mejor alternativa si lo que se desea es fabricar productos para revestimientos de paredes, que requieren un mayor control dimensional. Las perdidas de calcinación no presentan diferencias significativas entre un método y otro, solo que con la arcilla roja se obtiene una menor compactación que permite una mejor evaporación del agua durante la operación de secado, la absorción de agua como parámetro de control permite obtener mejores resultados con la técnica de extrusión, esto se pudo corroborar en los datos mostrados en las curvas de absorción de agua y contracciones en cocido donde se evidencia una mas rápida vitrificación de las probetas, hecho que en términos económicos se ve reflejado en un menor consumo energético y una menor cantidad de emisiones generadas por la combustión del carbón mineral principal combustible empleado en la zona.

\section{AGRADECIMIENTOS}

Los autores expresan su agradecimiento al Centro de Investigación de Materiales Cerámicos CIMAC de la Universidad Francisco de Paula Santander por el apoyo brindado para la realización de los ensayos.

\section{BIBLIOGRAFIA}

(1). Jeskar Ltda, Inventario Geológico Minero, Ambiental, Tecnológico y Empresarial de los Minerales no Energéticos del Norte de Santander. San José de Cúcuta, Colombia (2000).

(2). Sánchez, J. Diagnostico general del sector cerámico del área metropolitana de Cúcuta. Grupo de Investigación en Tecnología Cerámica GITEC. San José de Cúcuta. Universidad Francisco de Paula Santander. Facultad de ciencias Básicas (2009).

(3). Fernández Abajo, M. Manual sobre fabricación de baldosas, tejas y ladrillos [DISCO MAGNETICO]. Laboratorio Técnico Cerámico, Igualada, España (2000).

(4). Carrillo, M, Arevalo, I., Actualización de la información y puesta en marcha de una aplicación sistematizada para el sector cerámico del área metropolitana de san José de Cúcuta. Tesis de pregrado en Ingeniería de Producción Industrial. Universidad Francisco de Paula Santander. Facultad de Ingeniería (2008).

(5). Ferro Corporation, Análisis químico de diferentes arcillas del área metropolitana de Cúcuta. San José de Cúcuta, (2001).

(6). Universidad Industrial de Santander UIS, Grupo de Investigaciones en Minerales, Biohidrometalurgia y Ambiente, Análisis mineralógico de diferentes arcillas del área metropolitana de Cúcuta. Bucaramanga, (2008).

(7). Centro de Investigación de Materiales Cerámicos, Caracterización física química de arcillas de la zona metropolitana de Cúcuta. San José de Cúcuta, Universidad Francisco de Paula Santander (2009).

(8). A. M. Querol ViUalba. Aplicación del método de Pfefferkorn al control de la plasticidad en pastas de extrusión. Boletín de la Sociedad Española de Cerámica y Vidrio. Vol. 22, 2, 285-325 (1983).

(9). Norma Técnica Colombiana NTC 4321-3 baldosas cerámicas .parte 3. Método de ensayo para determinar la absorción de agua, porosidad aparente, densidad relativa aparente y densidad aparente. Bogota D.C. ICONTEC (2005).

(10). American Society For Testing And Materials. ASTM C326-03. Standard Test Method for Drying and Firing Shrinkages of Ceramic Whiteware Clays (2003). 
(11). J. L. Amorós, et al. Estudio de la operación de prensado de baldosas de gran formato utilizando la absorción de rayos X. Boletín de la Sociedad Española de Cerámica y Vidrio. Vol. 49, 4, 279 - 288 (2010).

(12). O. P. Rodríguez, et al. Estudio de correlación y variabilidad de los métodos de inmersión en mercurio y el método capacitivo resonante para determinar densidad aparente de material cerámico crudo. Boletín de la Sociedad Española de Cerámica y Vidrio. Vol. 50, 1, 5-12 (2011).

(13). J. M. Villora, et al. Influencia del procesamiento de materiales cerámicos en su comportamiento como adsorbente de iones en aguas industriales. Boletín de la Sociedad Española de Cerámica y Vidrio. Vol. 43, 1, 75-79 (2004).

Recibido: 11/04/2012

Aceptado: 17/10/2012 